\title{
Stability Evaluation of the SDRE Technique based on Java in a CubeSat Attitude and Orbit Control Subsystem
}

\author{
ALESSANDRO GERLINGER ROMERO \\ Space Mechanics and Contro Division \\ Brazilian National Institute for Spaece Research \\ Astronautas Avenue, 1758 - São José dos Campos \\ BRAZIL \\ LUIZ CARLOS GADELHA DE SOUZA \\ Engineering Center \\ Federal University of ABC \\ dos Estados Avenue - São Bernardo do Campo \\ BRAZIL
}

\begin{abstract}
In 2013, the STRaND (University of Surrey and Surrey Satellite Technology Ltd) and the PhoneSat (NASA) programs attracted the attention of the aerospace community applying commercial off-the-shelf smartphones in CubeSats. Both programs deployed CubeSats using smartphones based on Google's Android, in which application development is mainly based on Java programming language. Some of these CubeSats had actuators, e.g., STRaND-1 had three reaction wheels mounted in an orthogonal configuration to provide three-axis control, whereas PhoneSat 2.0 beta had magnetorquers to de-tumble the spacecraft. Taking into account a CubeSat that runs Android operating system (based on a smartphone), it is natural to evaluate the attitude and orbit control subsystem (AOCS) based on Java. Elsewhere, we shown State-Dependent Riccati Equation (SDRE) is a feasible non-linear control technique that can be applied in such CubeSats using Java. Moreover, we shown, through simulation using a Monte Carlo perturbation model, SDRE provides better performance than the PID controller, a linear control technique. In this paper, we tackle the next fundamental problem: stability. We evaluate stability from two perspectives: (1) parametric uncertainty of the inertia tensor and (2) a Monte Carlo perturbation model based on a uniform attitude probability distribution. Through the combination of these two perspectives, we grasp the stability properties of SDRE in a broader sense. In order to handle the uncertainty appropriately, we combine SDRE with $H_{\infty}$. The Nanosatellite Constellation for Environmental Data Collection (CONASAT), a CubeSat from the Brazilian National Institute for Space Research (INPE), provided the nominal parameters for the simulations. The initial results of the simulations shown that the SDRE controller is stable to $\pm 20 \%$ uncertainty in the inertia tensor for attitudes uniformly distributed and angular velocity up to 0.15 radians/second.
\end{abstract}

Key-Words: CubeSat, SDRE, $H_{\infty}$, nonlinear, control, attitude

Received: September 20, 2020. Revised: December 31, 2020. Accepted: January 25, 2021. Published: February 10, 2021.

\section{Introduction}

In 2013, the STRaND (University of Surrey and Surrey Satellite Technology Ltd) [1] and the PhoneSat (NASA) [2] programs attracted the attention of the aerospace community applying commercial off-the-shelf smartphones in CubeSats. Both programs deployed CubeSats using smartphones based on Google's Android, in which application development is mainly based on Java programming language. Some of these CubeSats had actuators, e.g., STRaND1 had three reaction wheels mounted in an orthogonal configuration to provide three-axis control, whereas PhoneSat 2.0 beta had magnetorquers to de-tumble the spacecraft. Taking into account a CubeSat that runs Android operating system (based on a smartphone), it is natural to evaluate the attitude and orbit control subsystem (AOCS) based on Java.

Furthermore, the design of a satellite attitude and orbit control subsystem (AOCS) that involves plant uncertainties, large angle maneuvers, and fast attitude control following a stringent pointing, requires nonlinear control methods in order to satisfy performance and robustness requirements.

Elsewhere, we shown State-Dependent Riccati Equation (SDRE) is a feasible non-linear control technique that can be applied in such CubeSats using Java [3]. Moreover, we shown, through simulation using a Monte Carlo perturbation model, SDRE pro- 
vides better performance than the PID controller, a linear control technique.

In this paper, we tackle the next fundamental problem: stability. We evaluate stability from two perspectives: (1) parametric uncertainty of the inertia tensor and (2) a Monte Carlo perturbation model based on a uniform attitude probability distribution. Through the combination of these two perspectives, we grasp the stability properties of SDRE in a broader sense. In order to handle the uncertainty appropriately, we combine SDRE with $H_{\infty}$.

SDRE was originally proposed by [4] and then explored in detail by [5]. A good survey of the SDRE method can be found in [6] and its systematic application to deal with a nonlinear plant in [7]. The SDRE method was applied by [8, 9, 10, 3, 11] for controlling a nonlinear system similar to the sixdegree of freedom satellite model considered in this paper. [8] defined a simulator using Euler angles based on commercial software, whereas, [9] applied quaternions on commercial software. [11] applied the SDRE as a filter technique together with a PID controller. [10, 3] shown, through simulation applying opensource software, using a Monte Carlo perturbation model, SDRE based on quaternions provides better performance than the PID controller.

SDRE method can be readily extended to nonlinear $H_{\infty}$ [5]. The interest in $H_{\infty}$ optimization for robust control of linear plants is mostly attributed to the influential work of [12], in which the problem of sensitivity reduction by feedback is formulated as an optimization problem. This problem can be numerically solved with the help of the well-known approaches based on Riccati equations or Linear Matrix Inequalities (LMIs) [13]. Later, [14] addressed the problem of robustly stabilizing a family of linear systems in the case where such family was characterized by $H_{\infty}$ bounded perturbations of a normalized left coprime factorization of a nominal system.

Nano satellite constellation for environmental data collection (CONASAT) [11], a CubeSat from the Brazilian National Institute for Space Research (INPE), provided the nominal parameters for the simulations. The initial results of the simulations shown that the SDRE with $H_{\infty}$ controller is stable to +$10 \%$ uncertainty in the inertia tensor for attitudes uniformly distributed and angular velocity up to 0.05 radians/second.

This paper is organized as follows. In Section 2 , the problem description is presented. In Section 3 , for the sake of completeness, the satellite physical modeling in the simulator is briefly reviewed. In Section 4 . we explore the state-space models and the controllers. In Section 5, we share simulation results. Finally, the conclusions are shared in Section 6 .

\section{Problem Description}

The SDRE technique entails factorization (that is, parametrization) of the nonlinear dynamics into the state vector and the product of a matrix-valued function that depends on the state itself. In doing so, SDRE brings the nonlinear system to a (nonunique) linear structure having state-dependent coefficient (SDC) matrices given by Eq. (1).

$$
\begin{array}{r}
\overrightarrow{\dot{x}}=A(\vec{x}) \vec{x}+B(\vec{x}) \vec{u} \\
\vec{y}=C(\vec{x}) \vec{x}
\end{array}
$$

where $\vec{x} \in \mathbb{R}^{n}$ is the state vector and $\vec{u} \in \mathbb{R}^{m}$ is the control vector. Notice that the SDC form has the same structure as a linear system, but with the system matrices, $A$ and $B$, being functions of the state vector. The nonuniqueness of the SDC matrices creates extra degrees of freedom, which can be used to enhance controller performance, however, it poses challenges since not all SDC matrices fulfill the SDRE requirements, e.g., the pair $(A, B)$ must be pointwise stabilizable.

The system model in Eq. (1) is subject of the cost functional described in Eq. (2).

$$
J(\vec{x}, \vec{u})=\frac{1}{2} \int_{0}^{\infty}\left(\vec{x}^{T} Q(\vec{x}) \vec{x}+\vec{u}^{T} R(\vec{x}) \vec{u}\right) d t
$$

where $Q(\vec{x}) \in \mathbb{R}^{n \times n}$ and $R(\vec{x}) \in \mathbb{R}^{m \times m}$ are the state-dependent weighting matrices. In order to ensure local stability, $Q(\vec{x})$ is required to be positive semi-definite for all $\vec{x}$ and $R(\vec{x})$ is required to be positive for all $\vec{x}[15]$.

The SDRE controller linearizes the plant about the current operating point and creates constant statespace matrices so that the LQR method can be used. This process is repeated in all samplings steps, resulting in a pointwise linear model from a non-linear model, so that an algebraic Riccati equation (ARE) is solved and a control law is computed also in each step. Therefore, according to LQR theory and Eq. (1) and (2), the state-feedback control law in each sampling step is $\vec{u}=-K(\vec{x}) \vec{x}$ and the state-dependent gain $K(\vec{x})$ is obtained by Eq. (3) [7].

$$
K(\vec{x})=R^{-1}(\vec{x}) B^{T}(\vec{x}) P(\vec{x})
$$

where $P(\vec{x})$ is the unique, symmetric, positivedefinite solution of the algebraic state-dependent Riccati equation (SDRE) given by Eq. (4) [7].

$$
\begin{array}{r}
P(\vec{x}) A(\vec{x})+A^{T}(\vec{x}) P(\vec{x}) \\
-P(\vec{x}) B(\vec{x}) R^{-1}(\vec{x}) B^{T}(\vec{x}) P(\vec{x}) \\
+Q(\vec{x})=0
\end{array}
$$

Considering that Eq. (4) is solved in each sampling step, it is reduced to an ARE. Finally, the conditions 
for the application of the SDRE technique in a given system model are [7]:

1. $A(\vec{x}) \in C^{1}\left(\mathbb{R}^{w}\right)$

2. $B(\vec{x}), C(\vec{x}), Q(\vec{x}), R(\vec{x}) \in C^{0}\left(\mathbb{R}^{w}\right)$

3. $Q(\vec{x})$ is positive semi-definite and $R(\vec{x})$ is positive definite

4. $A(\vec{x}) x \Longrightarrow A(0) 0=0$, i.e., the origin is an equilibrium point

5. $\operatorname{pair}(A, B)$ is pointwise stabilizable (a sufficient test for stabilizability is to check the rank of controllability matrix)

6. $\operatorname{pair}\left(A, Q^{\frac{1}{2}}\right)$ is pointwise detectable (a sufficient test for detectability is to check the rank of observability matrix)

\subsection{SDRE with $H_{\infty}$}

SDRE method can be readily extended to nonlinear $H_{\infty}$ [5]. See also [5] for stability concerns of such extension. Consider the general nonlinear dynamics using SDC as:

$$
\begin{array}{r}
\dot{x}=A(x) x+B_{1}(x) w+B_{2}(x) u \\
z=C_{1}(x) x+D_{12}(x) u \\
y=C_{2}(x) x+D_{21}(x) u
\end{array}
$$

where $\vec{x} \in \mathbb{R}^{n}$ is the state vector, $\vec{u} \in \mathbb{R}^{m}$ is the control vector, $\vec{y} \in \mathbb{R}^{q}$ is the output vector, $\vec{w} \in \mathbb{R}^{m}$ is the vector of exogenous signals (e.g., disturbances) and $\vec{z} \in \mathbb{R}^{n}$ is the vector of error signal which is to be minimized in some sense to meet control objectives. In such a way that the attention is moved to the size of error signals and away from the size and bandwidth of selected closed-loop transfer function. Furthermore, the additional functions are $C^{0}\left(\mathbb{R}^{w}\right)$.

Consider such state-space model, Eq. (5), described by a transfer function $G$. Now consider the stabilization of plant $G$ which has a normalized left coprime factorization [14, 16]:

$$
G=M^{-1} N
$$

then a perturbed plant model $G_{p}$ can be written as [16]:

$$
G_{p}=\left(M+\Delta_{M}\right)^{-1}\left(N+\Delta_{N}\right)
$$

where $\Delta_{M}, \Delta_{N}$ are stable unknown transfer functions which represent the uncertainty in the nominal plant G.

The objective of robust stabilization $H_{\infty}$ is to stabilize not only the nominal plant $G$, but a family of perturbed plants defined by [14, 16]:

$$
G_{p}=\left\{\left(M+\Delta_{M}\right)^{-1}\left(N+\Delta_{N}\right)::\right.
$$$$
\left.\left\|\left[\Delta_{M} \Delta_{N}\right]\right\|_{\infty}<\epsilon\right\}
$$

where $\epsilon>0$ is the stability margin, which is a measure of how close a stable closed-loop system is to instability. To maximize this stability margin is the problem of $H_{\infty}$ robust stabilization of normalized coprime factor plant descriptions [14].

For the positive feedback of Fig. 1, the perturbed plant is robustly stabilizable if and only if the nominal feedback is stable and [14, 16]

$$
\left\|\left[\begin{array}{c}
K \\
I
\end{array}\right](I-G K)^{-1} M^{-1}\right\|_{\infty} \leq \epsilon^{-1}
$$

The maximum stability margin $\epsilon$ and the corresponding lowest achievable value of $\gamma$ are given as [14]:

$$
\gamma_{\min }=\epsilon_{\max }^{-1}=(1+\rho(X Z))^{\frac{1}{2}}
$$

where $\rho$ denotes the spectral radius (maximum eigenvalue) and for the initial state-space realization $Z$ and $X$ are solutions of AREs.

$Z$ and $X$ are the solutions to the AREs [16, 14]:

$$
\begin{array}{r}
\left(A-B S^{-1} D^{T} C\right) Z+Z\left(A-B S^{-1} D^{T} C\right)^{T} \\
-Z C^{T} R^{-1} C Z+B S^{-1} B^{T}=0 \\
\left(A-B S^{-1} D^{T} C\right)^{T} x+X\left(A-B S^{-1} D^{T} C\right) \\
-X B S^{-1} B^{T} X+C^{T} R^{-1} C=0 \\
R=I+D D^{T} \\
R=I+D^{T} D
\end{array}
$$

A controller which guarantees that [14, 16]:

$$
\left\|\left[\begin{array}{c}
K \\
I
\end{array}\right](I-G K)^{-1} M^{-1}\right\|_{\infty} \leq \gamma
$$

for a specified $\gamma>\gamma_{m i n}$, is given by:

$$
\begin{array}{r}
K_{H_{\infty}}=\left[\begin{array}{rr}
A+B F+\gamma^{2}\left(L^{T}\right)^{-1} Z C^{T}(C+D F) & \gamma^{2}\left(L^{T}\right)^{-1} Z C^{T} \\
-D^{T}
\end{array}\right] \\
B^{T} X \quad F=-S^{-1}\left(D^{T} C+B^{T} X\right) \\
L=\left(1-\gamma^{2}\right) I+X Z
\end{array}
$$




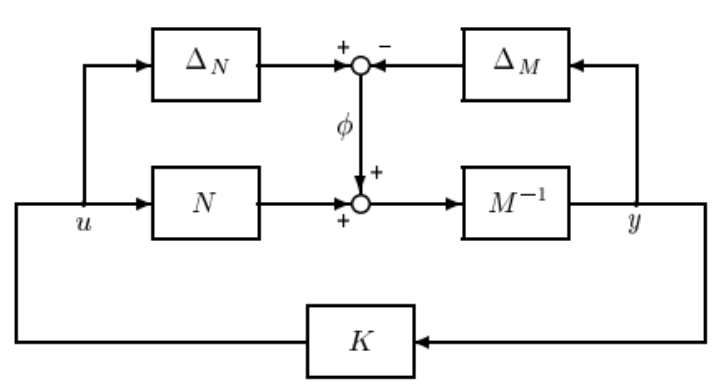

Figure 1: $H_{\infty}$ robust stabilization problem with left coprime factorization [16].

Therefore, regarding the combination of SDRE and $H_{\infty}$ the procedure to compute the controller that maximizes the stability margin for the perturbed plants in each step is:

1. Reconstruct the matrices using the SDC form;

2. Solve the two ARES of Eq. (11) computing $X$ and $Z$;

3. Compute $\gamma_{\min }$ using Eq. (10);

4. Define a state-space model (A,B,C,D) using $X$, $Z$ and a $\gamma>\gamma_{\min }$ by Eq. (13);

5. Solve the third ARE that results from statespace model described by Eq. (13), which leads to $P_{K_{H \infty}}$ as the unique, symmetric, positivedefinite solution of such ARE;

6. Compute the controller $K$ for the original system using $K(\vec{x})=R^{-1}(\vec{x}) B_{2}(\vec{x}) P_{K_{H_{\infty}}}(\vec{x})$.

It is known that if a controller can be found using that procedure, the exogenous signal will be locally attenuated by $\gamma[16,5,14]$ in each step.

\section{Satellite Physical Modeling}

A CubeSat, e.g., STRaND-1 [1] and CONASAT [11], can be stabilized in three-axes so that the payload can point to the desired target. Next subsections explore the kinematics and the rotational dynamics of a CubeSat in analysis.

\subsection{Kinematics}

Given the ECI reference frame $\left(\mathfrak{F}_{i}\right)$ and the frame defined in the satellite with origin in its centre of mass (the body-fixed frame, $\mathfrak{F}_{b}$ ), then a rotation represented

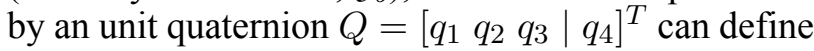
the attitude of the satellite.
Defining the angular velocity $\vec{\omega}=\left[\omega_{1} \omega_{2} \omega_{3}\right]^{T}$ of $\mathfrak{F}_{b}$ with respect to $\mathfrak{F}_{i}$ measured in the $\mathfrak{F}_{b}$, the kinematics can be described by Eq. (14) [17].

$$
\begin{array}{r}
\dot{Q}=\frac{1}{2} \Omega(\vec{\omega}) Q=\frac{1}{2} \Xi(Q) \vec{\omega} \\
\Omega(\vec{\omega}) \triangleq\left[\begin{array}{cccc}
0 & \omega_{3} & -\omega_{2} & \omega_{1} \\
-\omega_{3} & 0 & \omega_{1} & \omega_{2} \\
\omega_{2} & -\omega_{1} & 0 & \omega_{3} \\
-\omega_{1} & -\omega_{2} & -\omega_{3} & 0
\end{array}\right],
\end{array}
$$

where the unit quaternion $Q$ satisfies the identity: $q_{1}^{2}+$ $q_{2}^{2}+q_{3}^{2}+q_{4}^{2}=1$.

Eq. (14) allows the prediction of the satellite's attitude if it is available the initial attitude and the history of the change in the angular velocity $(\dot{Q}=F(\omega, t))$.

Another possible derivation of the Eq. (14) is using the vector $\mathrm{g}$ (Gibbs vector or Rodrigues parameter) as $Q=\left[g^{T} \mid q_{4}\right]$.

$$
\dot{Q}=-\frac{1}{2}\left[\begin{array}{c}
\omega^{\times} \\
\omega^{T}
\end{array}\right]\left[\begin{array}{l}
q_{1} \\
q_{2} \\
q_{3}
\end{array}\right]+\frac{1}{2} q_{4}\left[\begin{array}{c}
1_{3 \times 3} \\
0
\end{array}\right] \vec{\omega}
$$

where $\omega^{\times}$is the cross-product skew-symmetric matrix of $\vec{\omega}$ and 1 is the identity matrix. Note the Gibbs vector is geometrically singular since it is not defined for $180^{\circ}$ of rotation [18], nonetheless, the Eq. (15) is global.

\subsection{Rotational Dynamics}

In order to know the history of the change in the angular velocity, it is necessary to understand the history of the change in the angular acceleration $(\dot{\vec{\omega}}=G(\tau, t))$ of the satellite. According to the Euler-Newton formulation of the rotational motion, angular acceleration is caused by torques, in other words, the change in the angular momentum $\vec{h}$ is equal to the net torques $\vec{g}$ applied in the satellite, see Eq. (16) (the present subsection is derived based on the centre of mass of the satellite, for the general case, see [17]).

$$
\overrightarrow{\dot{h}}=\vec{g}
$$

The angular momentum is also known as the moment of momentum since it defines the moment of a given momentum $\vec{p}(\vec{p} \triangleq m \vec{v})$ about a given point $P_{c m}$. See Eq. (17), in which $r$ locates a given point $p$ with respect to $P_{c m}$.

$$
\vec{h}=\vec{r} \times \vec{p}
$$

Taking into account the motion of the body-fixed frame $\mathfrak{F}_{b}$ with respect to the ECI $\mathfrak{F}_{i}$ and the angular 
velocity $\vec{\omega}$, the derivative of the angular momentum in $\mathfrak{F}_{b}$ is defined by Eq. (18).

$$
\overrightarrow{\dot{h}}=\vec{g}-\vec{\omega} \times \vec{h}
$$

Furthermore, $\overrightarrow{\dot{h}}=I . \overrightarrow{\dot{\omega}}$ and $\vec{h}=I . \vec{\omega}$, where $I$ is the time-invariant inertia tensor. The combination of this definition and Eq. (18) results in Eq. (19).

$$
I . \overrightarrow{\dot{\omega}}=\vec{g}-\vec{\omega} \times(I . \vec{\omega})
$$

The CubeSats in analysis have a set of 3 reaction wheels, each one aligned with its principal axes of inertia, moreover, such type of actuator, momentum exchange actuators, does not change the angular momentum of the satellite. Consequently, it is mandatory to model their influence in the satellite, in particular, the angular momentum of the satellite is defined by Eq. (20).

$$
\vec{h}=\left(I-\sum_{n=1}^{3} I_{n, s} a_{n} a_{n}^{T}\right) \vec{\omega}+\sum_{n=1}^{3} h_{w, n} \overrightarrow{a_{n}}
$$

where $I_{n, s}$ is the inertia moment of the reaction wheels in their symmetry axis $\overrightarrow{a_{n}}, h_{w, n}$ is the angular momentum of the $n$ reaction wheel about its centre of mass $\left(h_{w, n}=I_{n, s} a_{n}^{T} \omega+I_{n, s} \omega_{n}\right)$ and $\omega_{n}$ is the angular velocity of the $n$ reaction wheel.

One can define $I_{b}$ using Eq. (21).

$$
I_{b}=I-\sum_{n=1}^{3} I_{n, s} a_{n} a_{n}^{T}
$$

Using $I_{b}$, the motion of the satellite is described by Eq. (22) .

$$
\begin{array}{r}
\dot{\vec{\omega}}^{b}=I_{b}^{-1} \overrightarrow{g_{c m}}-I_{b}^{-1} \omega^{\times} I_{b} \vec{\omega} \\
-I_{b}^{-1} \omega^{\times} \sum_{n=1}^{3} h_{w, n} \overrightarrow{a_{n}}-I_{b}^{-1} \sum_{n=1}^{3} g_{n} \overrightarrow{a_{n}}
\end{array}
$$

where $g_{c m}$ is the net external torque and $g_{n}$ are the torques generated by the reactions wheels $\left(\dot{h_{w, n}}=\right.$ $\left.g_{n}\right)$.

\section{Controller Design}

In a zero-bias-momentum system, two dynamics states must be controlled: (1) the attitude (perhaps described by unit quaternions $Q$ ) and (2) its stability $(\dot{Q}$, in other words, the angular velocity $\omega$ of the satellite).
The state and the control vectors, for the control loop, can be defined by Eq. (23).

$$
\left[\begin{array}{l}
\overrightarrow{x_{1}} \\
\overrightarrow{x_{2}}
\end{array}\right]=\left[\begin{array}{l}
Q \\
\vec{\omega}
\end{array}\right]
$$

$$
\left[\overrightarrow{u_{1}}\right]=\left[\sum_{n=1}^{3} g_{n} \overrightarrow{a_{n}}\right]
$$

The control regulator problem requires that the errors in the attitude and angular velocity must be obtained. The error in the angular velocity is directly obtained from the gyroscope readings, nonetheless, the error in the attitude must be computed. The applied approach to computation of the error in the attitude is: given two versors, namely (a) the actual Sun versor $s_{b}$ in the satellite frame obtained by the proper transformation of the Sun versor in the ECI $\left(s_{i}\right)$; and (b) the reference versor in the satellite frame; to compute a rotation (there are many) from the actual Sun versor to the reference versor. The computed rotation can be described by an unit quaternion $Q$.

\subsection{Nonlinear Control based on State-Dependent Riccati Equation (SDRE) Controller}

Assuming that there are no net external torques $\left(g_{c m}=0\right)$, the rotational dynamics defined in Eq. (22) can be rearranged as defined by Eq. (24) (applying the property $v^{\times} w=-w^{\times} v$ ). Eq. (24) is relevant since it is applied in the state-space formulation using the state and control vectors in Eq. (23).

$$
\begin{array}{r}
\dot{\vec{\omega}}=\left(-I_{b}^{-1} \omega^{\times} I_{b}+I_{b}^{-1}\left(\sum_{n=1}^{3} h_{w, n} a_{n}\right)^{\times}\right) \vec{\omega} \\
-I_{b}^{-1} \sum_{n=1}^{3} g_{n} a_{n}
\end{array}
$$

Taking into account the state and control vectors defined in Eq. (23), the state-space model can be defined using Eq. (14) $(\Omega)$ and Eq.(24). However, the SDC matrices do not fulfill the SDRE requirements, in particular, the pair $(\mathrm{A}, \mathrm{B})$ is not pointwise stabilizable. An alternative option for the definition of the SDC matrices is to use Eq. (15) (Gibbs vector or Rodrigues parameter) and Eq.(24), which leads to Eq. (25). Eq. (25) has been shown to satisfy SDRE conditions described in Section 2 .

$$
\begin{array}{r}
{\left[\begin{array}{c}
\dot{x_{1}} \\
\dot{x_{2}}
\end{array}\right]=\left[\begin{array}{ccc}
-\frac{1}{2}\left[\begin{array}{c}
\omega^{\times} \\
\omega^{T}
\end{array}\right] & 0 & {\left[\begin{array}{c}
\frac{1}{2} q_{4} I_{3 \times 3} \\
0 \\
0
\end{array}\right.} \\
0 & -I_{b}^{-1} \omega^{\times} I_{b}+I_{b}^{-1}\left(\sum_{n=1}^{3} h_{w, n} a_{n}\right)^{\times}
\end{array}\right]\left[\begin{array}{l}
x_{1} \\
x_{2}
\end{array}\right]+\left[\begin{array}{c}
0 \\
-I_{b}^{-1}
\end{array}\right]\left[u_{1}\right]} \\
{[y]=I\left[\begin{array}{l}
x_{1} \\
x_{2}
\end{array}\right]}
\end{array}
$$


Table 1: Satellite characteristics and references.

\begin{tabular}{c|c}
\hline Name & Value \\
\hline Satellite Characteristics & \\
\hline inertia tensor $\left(\mathrm{kg} \cdot \mathrm{m}^{2}\right)$ & $\operatorname{diag}(0.0547$, \\
& $0.0519,0.0574)$ \\
\hline Actuators Characteristics - Reaction Wheels \\
\hline inertia $\left(\mathrm{kg} \cdot \mathrm{m}^{2}\right)$ & 0.00015 \\
max torque $(N . \mathrm{m})$ & 0.000625 \\
max ang velocity $(R P M)$ & 750 \\
\hline References for the controller \\
\hline \multicolumn{2}{c}{ solar vector body $(\mathrm{XYZ})$} \\
angular velocity $(\mathrm{r} / \mathrm{s}, \mathrm{XYZ})$ & {$\left[\begin{array}{lll}1 & 0 & 0\end{array}\right]^{T}$} \\
\hline
\end{tabular}

\subsection{Nonlinear Control based on State-Dependent Riccati Equation (SDRE) with $H_{\infty}$ Controller}

Although the SDRE with $H_{\infty}$ controller uses the Eq. (25), it follows the procedure defined in Subsection 2.1. Such procedure requires the solving of three AREs in each step, instead of one ARE as usual in the SDRE controller.

\section{Simulation Results}

Table 1 shows the satellite characteristics [11] and references used in the simulation results.

In order to compare the performance of the controllers, a simulation test was conducted with the full Monte Carlo perturbation model described as follows: (1) the initial Euler angles of the nonlinear spacecraft system are randomly selected using independent uniform distributions (minimum $=$ $-180^{\circ}$, maximum $=180^{\circ}$ ); and (2) the initial angular velocity errors are randomly selected using independent uniform distributions (minimum $=$ $-0.15 \mathrm{rad} / \mathrm{s}$, maximum $=0.15 \mathrm{rad} / \mathrm{s}$ ). Moreover, in order to evaluate the robustness of the controller defined using SDRE with $H_{\infty}$, another Monte Carlo perturbation model is defined as follows: (1) each diagonal element of the inertia tensor defined in Table 1 is changed accordingly a uniform distribution $\pm 20 \%$.

The Monte Carlo model ran 10 times and in each time one simulation of the two controllers were executed. Such executions used simulation time 200 seconds, fixed step 0.005 seconds, the data presented in Table 1 and the following controllers, both defined by Eq. (25) and (3): (1) SDRE controller $(R=1$ and $Q=1)$ and (2) SDRE $+H_{\infty}$ controller $(R=1$ and $Q=1)$.

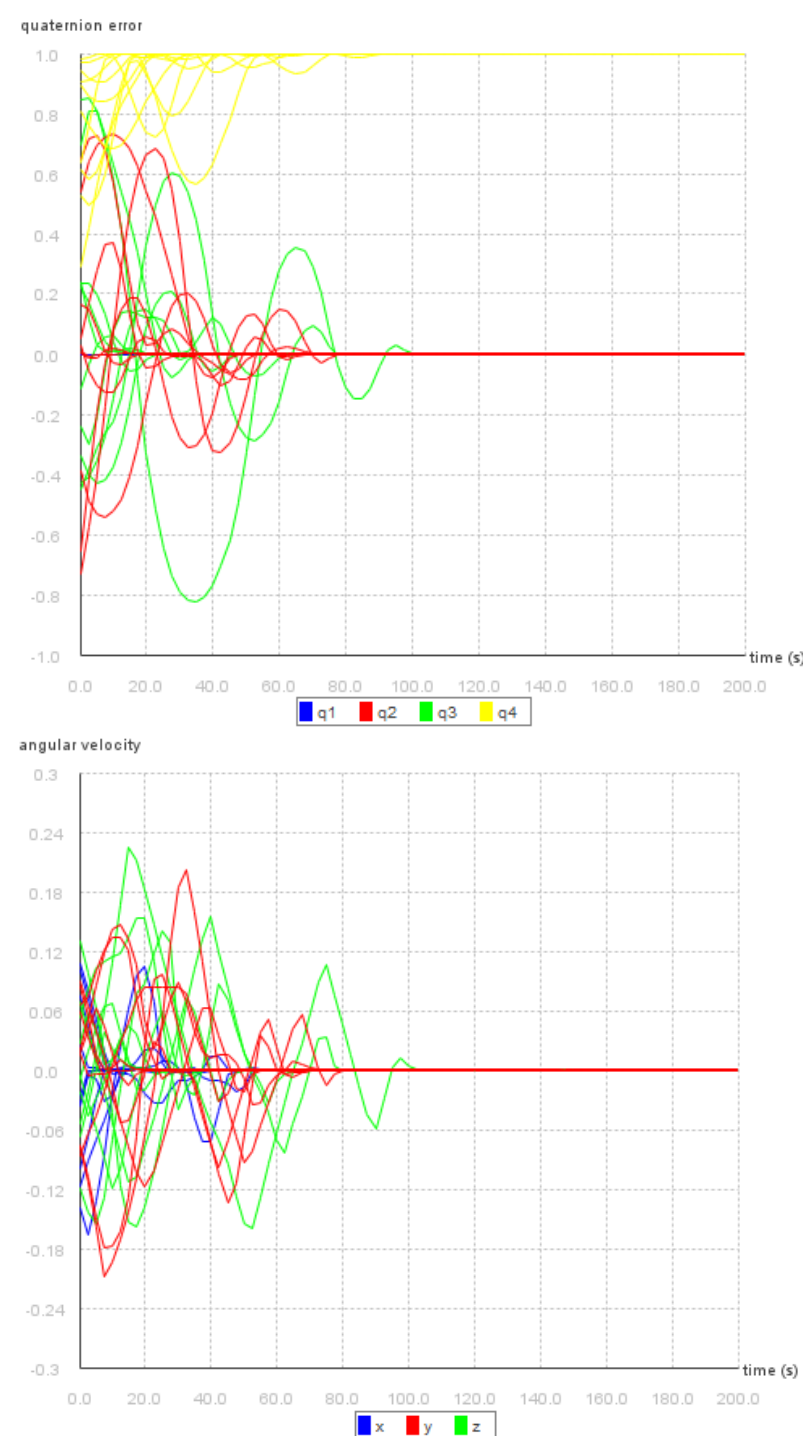

Figure 2: SDRE Gibbs Controller.

Each graph in Fig. 2 and Fig. 3 shows the respective collection of quaternion errors and angular velocities computed during simulations for a given controller. The SDRE $+H_{\infty}$ controller, Fig 3 , has the best performance since it uses less time to stabilize the initial conditions defined by the Monte Carlo perturbation model.

The Monte Carlo model ran 30 times using the same simulation parameters described above. The simulation results are shown in Fig. 4 . Such results are in accordance with Section 2 .

\section{Conclusion}

The major contribution of the current paper is the extension of SDRE with $H_{\infty}$ using exactly three AREs to find the sub-optimal controller, whereas the literature suggests the $\gamma$-iteration in each step in order to solve the general $H_{\infty}$ problem [5]. 

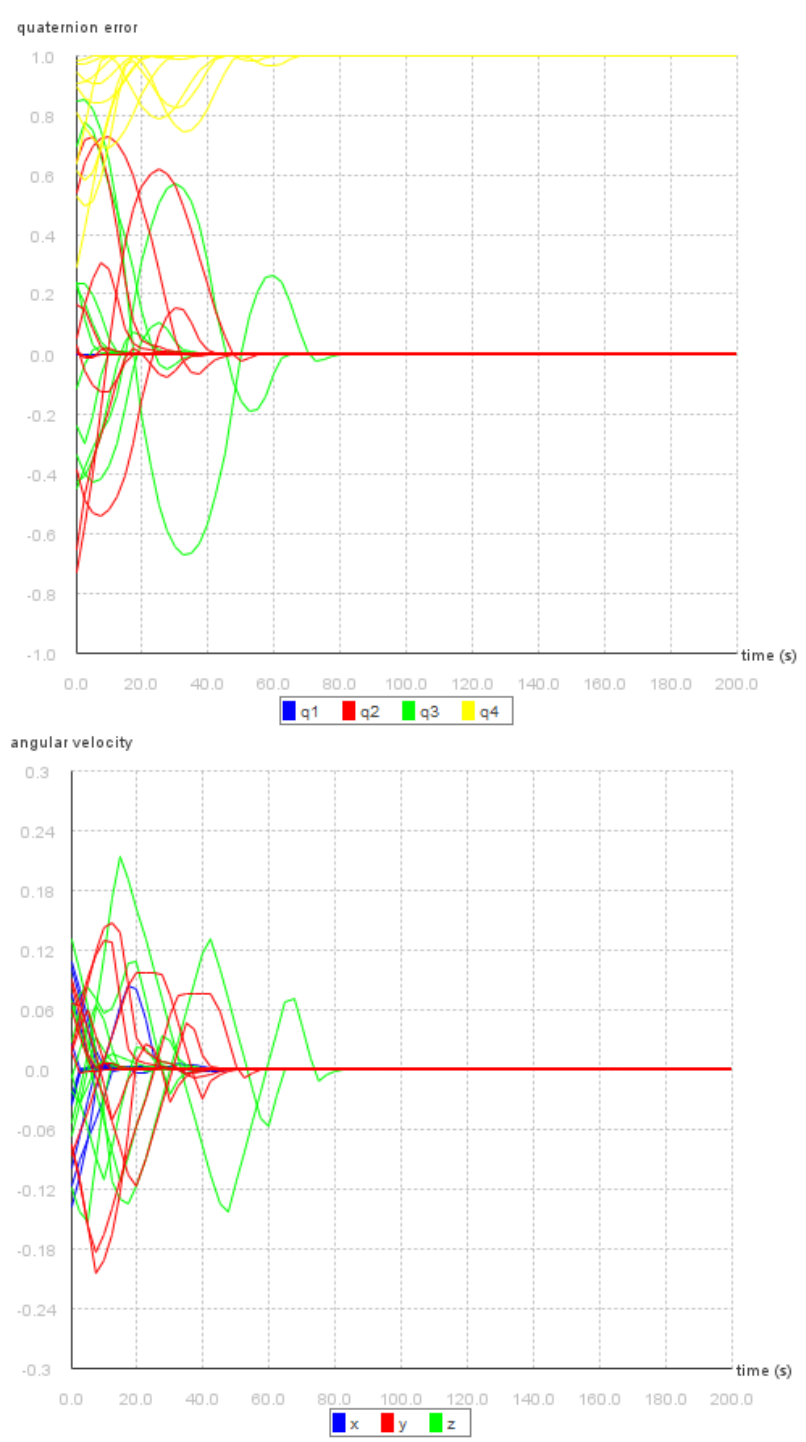

Figure 3: SDRE Gibbs $+H_{\infty}$ Controller.
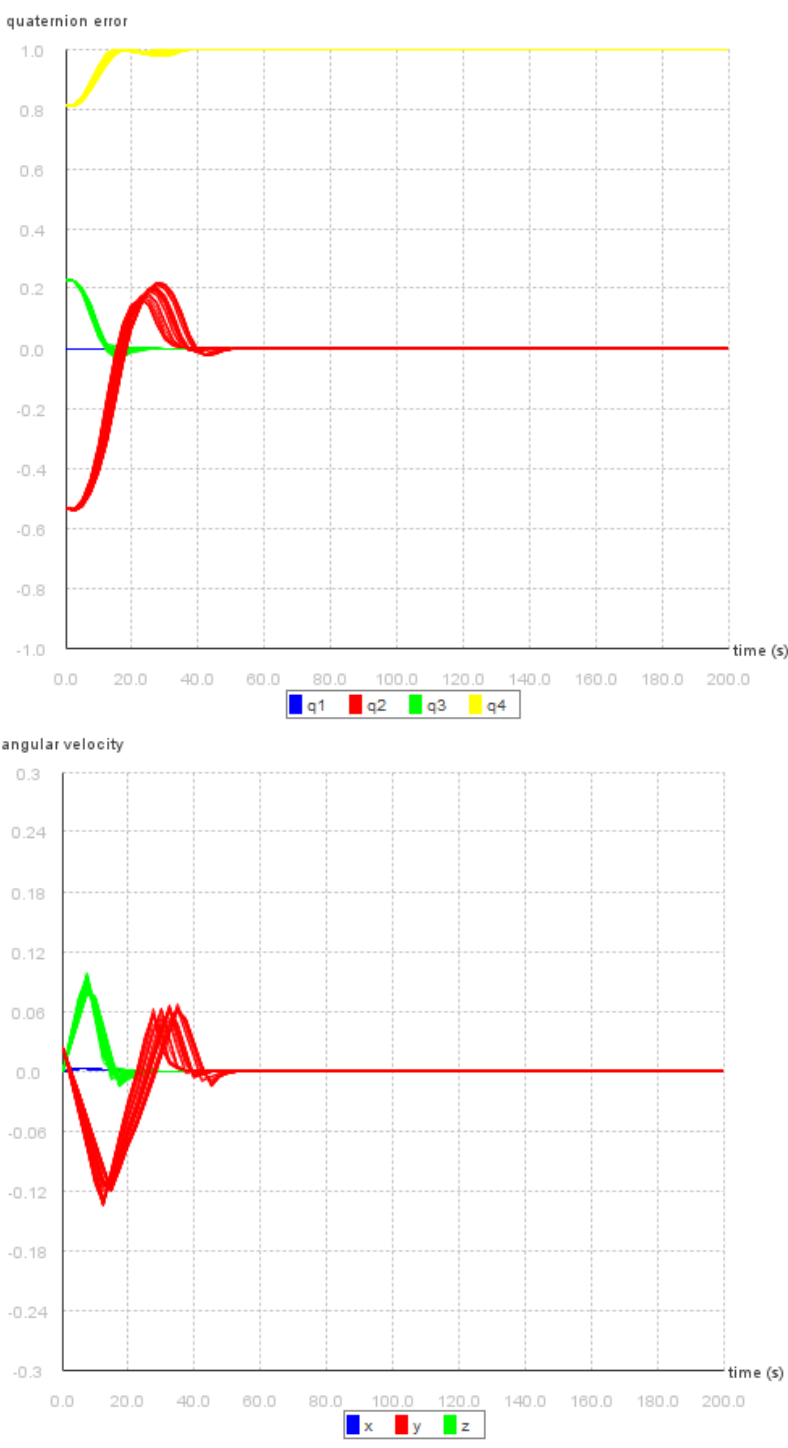

Figure 4: Simulation results for parametric uncertainty of SDRE Gibbs $+H_{\infty}$. 
There are two enhancements planned in the short term: (1) to run more tries of the Monte Carlo perturbation models; and (2) in a complement of the qualitative analysis showed in Fig. 2 and Fig. 3, to compute the standard deviation of the norm of the vectorial part of the quaternion error as well as the standard deviation of the norm of the angular velocity since the focus is on the regulator problem in which such quantities should converge to zero.

\section{References:}

[1] C Bridges, S Kenyon, C Underwood, and V Lappas. STRaND-1: The world's first smartphone nanosatellite. In 2011 2nd International Conference on Space Technology, pages 1--3, 2011.

[2] J. Gozalvez. Smartphones sent into space [mobile radio]. IEEE Vehicular Technology Magazine, 8(3):13--18, Sept 2013.

[3] Alessandro Gerlinger Romero. Satellite simulation developer's guide - attitude dynamics and control of nonlinear satellite simulations. Instituto Nacional de Pesquisas Espaciais, São José dos Campos, 2020.

[4] J. D. Pearson. Approximation Methods in Optimal Control I. Sub-optimal Control†. Journal of Electronics and Control, 13(5):453--469, 1962.

[5] James R Cloutier, Christopher N D'Souza, and Curtis P Mracek. Nonlinear regulation and nonlinear H-infinity control via the state-dependent Riccati equation technique. Conference on Nonlinear Problems in Aviation, (September), 1996.

[6] Tayfun Çimen. State-Dependent Riccati Equation (SDRE) control: A survey. IFAC Proceedings Volumes (IFAC-PapersOnline), 17(1 PART 1):3761--3775, 2008.

[7] Tayfun Çimen. Systematic and effective design of nonlinear feedback controllers via the statedependent Riccati equation (SDRE) method. Annual Reviews in Control, 34(1):32--51, 2010.

[8] R G Gonzales and Luiz Carlos Gadelha de Souza. Application of the sdre method to design a attitude control system simulator. $A d-$ vances in the Astronautical Sciences, 134(Part 1-3):2251--2258, 2009. Setores de Atividade: Educação.

[9] G Di Mauro, M Schlotterer, S Theil, and M Lavagna. Nonlinear Control for Proximity Operations Based on Differential Algebra. Journal of Guidance, Control, and Dynamics, 38(11):2173--2187, apr 2015.

[10] Alessandro Gerlinger Romero and Luiz Carlos Gadelha de Souza. Satellite controller system based on reaction wheels using the statedependent riccati equation (sdre) on java. In Katia Lucchesi Cavalca and Hans Ingo Weber, edi- tors, Proceedings of the 10th International Conference on Rotor Dynamics -- IFToMM, pages 547--561, Cham, 2019. Springer International Publishing.

[11] B. D. Reis de Mesquita and H. Koiti Kuga and V. Carrara. Estimation and Attitude Control in CONASAT Nominal Operation Mode: An Approach for SDRE Filter and PID Control. IEEE Latin America Transactions, 15(5):835--842, 2017.

[12] G. Zames. Feedback and optimal sensitivity: Model reference transformations, multiplicative seminorms, and approximate inverses. IEEE Transactions on Automatic Control, 26(2):301--320, April 1981.

[13] E. Veremey and Margarita Sotnikova. Spectral approach to ho-optimal siso synthesis problem. WSEAS Transactions on Systems and Control, 9:405--414, 012014.

[14] K. Glover and D. McFarlane. Robust stabilization of normalized coprime factor plant descriptions with $\mathrm{h} /$ sub infinity /-bounded uncertainty. IEEE Transactions on Automatic Control, 34(8):821--830, Aug 1989.

[15] P. K. Menon, T. Lam, L. S. Crawford, and V. H.L. Cheng. Real-time computational methods for SDRE nonlinear control of missiles. Proceedings of the American Control Conference, 1:232--237, 2002.

[16] Sigurd Skogestad and Ian Postlethwaite. Multivariable Feedback Control: Analysis and Design. John Wiley \& Sons, Inc., Hoboken, NJ, USA, 2005.

[17] Peter C Hughes. Spacecraft Attitude Dynamics. New York, 1986.

[18] Peter W. Fortescue and Graham G. Swinerd. Attitude Control. Spacecraft Systems Engineering, (June):289--326, 2011.

\section{Contribution of individual authors to the creation of a scientific article (ghostwriting policy)}

Alessandro Gerlinger Romero carried out the modeling, the code developing, the simulation and the writing.

Luiz Carlos Gadelha de Souza oriented the first author and contributed in the writing.

\section{Creative Commons Attribution License 4.0 (Attribution 4.0 International, CC BY 4.0)}

This article is published under the terms of the Creative Commons Attribution License 4.0

https://creativecommons.org/licenses/by/4.0/deed.en_US 